\title{
A Bright Fast Radio Burst from FRB 20200120E with Sub-100 Nanosecond Structure
}

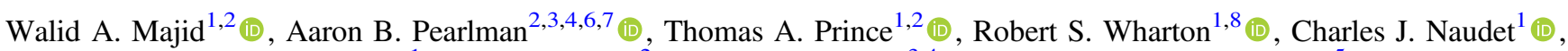 \\ Karishma Bansal $^{1}$ (D) Liam Connor $^{2}$ (D), Mohit Bhardwaj ${ }^{3,4}$ (D), and Shriharsh P. Tendulkar ${ }^{5}$ (D) \\ ${ }^{1}$ Jet Propulsion Laboratory, California Institute of Technology, Pasadena, CA 91109, USA; walid.majid@jpl.nasa.gov \\ ${ }^{2}$ Division of Physics, Mathematics, and Astronomy, California Institute of Technology, Pasadena, CA 91125, USA \\ ${ }^{3}$ Department of Physics, McGill University, 3600 rue University, Montréal, QC H3A 2T8, Canada \\ ${ }^{4}$ McGill Space Institute, McGill University, 3550 rue University, Montréal, QC H3A 2A7, Canada \\ ${ }^{5}$ Department of Astronomy and Astrophysics, Tata Institute of Fundamental Research, Mumbai, 400005 , India \\ Received 2021 May 29; revised 2021 July 21; accepted 2021 July 29; published 2021 September 22
}

\begin{abstract}
We have detected a bright radio burst from FRB 20200120E with the NASA Deep Space Network (DSN) $70 \mathrm{~m}$ dish (DSS-63) at radio frequencies between 2.2 and $2.3 \mathrm{GHz}$. This repeating fast radio burst (FRB) is reported to be associated with a globular cluster in the M81 galactic system. With high time resolution recording, low scattering, and large intrinsic brightness of the burst, we find a burst duration of $\sim 30 \mu \mathrm{s}$, comprised of several narrow components with typical separations of $2-3 \mu \mathrm{s}$. The narrowest component has a width of $\lesssim 100 \mathrm{~ns}$, which corresponds to a light travel time size as small as $30 \mathrm{~m}$. The peak flux density of the narrowest burst component is $270 \mathrm{Jy}$. We estimate the total spectral luminosity of the narrowest component of the burst to be $4 \times 10^{30} \mathrm{erg} \mathrm{s}^{-1}$ $\mathrm{Hz}^{-1}$, which is a factor of $\sim 500$ above the luminosities of the so-called "nanoshots" associated with giant pulses from the Crab pulsar. This spectral luminosity is also higher than that of the radio bursts detected from the Galactic magnetar SGR 1935 + 2154 during its outburst in April 2020, but it falls on the low-end of the currently measured luminosity distribution of extragalatic FRBs, further indicating the presence of a continuum of FRB luminosities. The temporal separation of the individual components has similarities to the quasiperiodic behavior seen in the microstructure of some pulsars. The known empirical relation between the microstructure quasiperiodicity timescale and the rotation period of pulsars possibly suggests a possible pulsar as the source of this FRB, with a rotation period of a few milliseconds.
\end{abstract}

Unified Astronomy Thesaurus concepts: Radio transient sources (2008); Radio bursts (1339)

\section{Introduction}

Fast radio bursts (FRBs) are energetic, short duration radio transients that are highly dispersed, with dispersion measures (DMs) that are well in excess of the expected Galactic contribution along their lines of sight (see, e.g., Cordes \& Chatterjee 2019; Petroff et al. 2019 for recent reviews). The localization of a subset of FRBs to host galaxies at redshifts of 0.034-0.66 has confirmed the extragalactic nature of FRBs (Chatterjee et al. 2017; Bannister et al. 2019; Prochaska et al. 2019; Ravi et al. 2019; Marcote et al. 2020). The current census of FRBs includes over 100 sources (Petroff et al. 2016), ${ }^{9} 24$ of which have been found to produce repeat bursts (e.g., see Spitler et al. 2016; The CHIME/FRB Collaboration et al. 2019a, 2019b; Fonseca et al. 2020). Despite the increasing number of FRB detections, their origin remains an open question with numerous progenitor and emission models that have been proposed to explain the nature of these mysterious bursts (see, e.g., Platts et al. $2019{ }^{10}$ for a catalog of theories and proposed models). Follow-up studies of repeaters with broadband instruments at higher time and frequency resolution as well as detailed polarimetric studies of repeating bursts are critical for constraining the progenitor models and for understanding of the FRB phenomenon (see, e.g., Nimmo et al. 2021a, 2021b).

\footnotetext{
${ }^{6}$ McGill Space Institute (MSI) Fellow.

7 FRQNT Postdoctoral Fellow.

8 NASA Postdoctoral Program Fellow.

9 See https://www.herta-experiment.org/frbstats/catalogue.

${ }^{10}$ See http://frbtheorycat.org.
}

FRB 20200120E is a repeating FRB, recently discovered by the Canadian Hydrogen Intensity Mapping Experiment Fast Radio Burst (CHIME/FRB) instrument (Bhardwaj et al. 2021). FRB 20200120E has the lowest reported dispersion measure (DM) to date among FRBs $\left(87.82 \mathrm{pc} \mathrm{cm}^{-3}\right)$ and very recently has been reported to be associated with a globular cluster in the M81 spiral galaxy system at a distance of $3.6 \mathrm{Mpc}$ (Kirsten et al. 2021a). If this association is correct, FRB 20200120E would be the nearest extragalactic FRB. At a distance that is $\sim 40$ times closer than the next closest FRB at $149 \mathrm{Mpc}$ (Marcote et al. 2020), FRB 20200120E can be used to explore burst luminosities $\gtrsim 10^{3}$ times lower than other FRBs.

Multifrequency follow-up studies are critical for characterizing FRB sources and emission models. One such approach is to carry out simultaneous observations of FRBs across wide bandwidths (Scholz et al. 2017, 2020; Majid et al. 2020; Pearlman et al. 2020) that are more robust against temporal evolution of scintillation and scattering, as well as intrinsic short term variability in the emission spectrum of individual bursts. Another approach is to probe the shorter timescales of the bursts to place limits on the instantaneous size of the emitting regions, similar to the studies of the Crab giant pulses carried out with few nanosecond time resolution (Hankins et al. 2003). Observations carried out at higher radio frequencies are particularly robust against temporal scattering due to multipath propagation that can limit the overall effective time resolution.

In this Letter, we present results from a simultaneous observation of FRB 20200120E at 2.25 and $8.36 \mathrm{GHz}$ with the NASA Deep Space Network (DSN) $70 \mathrm{~m}$ telescope, DSS-63, located in Madrid, Spain. The observation and data analysis 
procedures are described in Section 2. In Section 3, we provide measurements of the brightest burst detected, including the DM, width, flux density, fluence, and burst morphology. In Section 4, we discuss our measurements of the burst spectra, implications of the narrow components, burst energetics, and the impact of intrinsic and extrinsic effects on the burst properties.

\section{Observations and Data Analysis}

We observed FRB 20200120E continuously for $4.1 \mathrm{hr}$, starting at 2021 March 2 21:35:20 UTC (MJD 59275.89953), using DSS63 , the NASA DSN $70 \mathrm{~m}$ radio telescope located at the Madrid Deep Space Communication Complex (MDSCC) in Robledo, Spain. This observation was performed using the pointing position R.A. $(J 2000)=09: 57: 56.7$, decl. $(J 2000)=+68: 49: 31.8$. We carried out this observation as part of a monitoring program of repeating FRBs at high frequencies with the DSN's large $70 \mathrm{~m}$ radio telescopes, making use of the available gap times in the DSN's schedule for radio astronomical observation of FRBs. DSS-63 is equipped with cryogenically cooled, dual circular polarization receivers, which are capable of recording data simultaneously at S-band and X-band The center frequencies of the recorded S-band and X-band data were 2.25 and $8.36 \mathrm{GHz}$, respectively. The S-band system has an effective bandwidth of $115 \mathrm{MHz}$, after taking into account the front-end filter roll-off and masking bad channels contaminated by radio frequency interference (RFI). The X-band receivers provide $450 \mathrm{MHz}$ of usable bandwidth. Data from both polarization channels were simultaneously received and recorded at each frequency band with two different recorders at the site's Signal Processing Center. One of the recorders is the wide-band pulsar machine, capable of recording four independent input bands simultaneously, each with a bandwidth of $500 \mathrm{MHz}$ with a frequency resolution of $0.5 \mathrm{MHz}$ and time resolution of $2.2 \mathrm{~ms}$. In addition, we used a set of baseband recorders to record both S-band and X-band data in two polarization modes with time resolution of $62.5 \mathrm{~ns}$, albeit at the expense of sacrificing some portion of the available bandwidth. The analysis described in this Letter used data from the baseband recorders, and as such we will describe the configuration of these recorders in some detail below. The three baseband recorders were configured with a variable number of $16 \mathrm{MHz}$ sub-bands, with two-bit per sample recording. One recorder was used to record both polarization channels at S-band, with seven sub-bands for each polarization channel, spanning 2197-2309 MHz contiguously. The second and third baseband recorders were used to record the two polarization channels at $\mathrm{X}$-band, using 16 sub-bands for each polarization channel, spanning 8200-8456 MHz.

The initial data processing procedures were similar to those described in previous studies of the Crab giant pulses with the DSN (e.g., Majid et al. 2011). A nominal DM value of $87.82 \mathrm{pc} \mathrm{cm}^{-3}$, as initially reported by Bhardwaj et al. (2021), was used to coherently dedisperse the data in each sub-band. The power in each sub-band's time series was summed over all sub-bands to form one time series per polarization mode. A list of burst candidates with detection signal-to-noise ratios $(\mathrm{S} / \mathrm{N})$ above 7.0 were generated using a matched filtering algorithm, in which each dedispersed time series was convolved with boxcar functions ranging in widths from $62.5 \mathrm{~ns}$ up to $16.2 \mathrm{~ms}$, in multiplicative factors of 2 . For the bright burst described in this Letter, we subsequently extracted a few seconds of data around the nominal burst time and coherently dedispersed that data over a range of DM values to obtain an optimal DM value of $87.77(2) \mathrm{pc} \mathrm{cm}^{-3}$ while maximizing the $\mathrm{S} / \mathrm{N}$ of the burst. Using the updated DM value, we then proceeded to correct the data for the bandpass slope across the frequency band. We also subtracted the moving average from each data value using $0.5 \mathrm{~s}$ around each time sample to remove the low frequency temporal variability. The data samples were then normalized by the offburst standard deviation, before summing the two polarization bands to form total power spectra.

The data in each observing band were flux calibrated using nominal $T_{\text {sys }}$ values published by the DSN, ${ }^{11}$ which we previously verified via $\mathrm{ON}-\mathrm{OFF}$ observations of bright $3 \mathrm{C}$ calibrators, such as $3 \mathrm{C} 123,3 \mathrm{C} 48$, and Cas A. The $T_{\text {sys }}$ values were corrected for elevation effects, which are minimal for elevations greater than 20 degrees. We conservatively estimate the error on flux densities in each band to be $15 \%$, or less.

\section{Results}

We detected several bursts from FRB 20200120E during this $4.1 \mathrm{hr}$ observation. Among these, one burst stood out as the brightest with very detailed structure down to the native time resolution of the observation. In this Letter, we describe the properties of this burst, hereafter referred to as B1, and defer the presentation of the remaining bursts and their attributes to a later publication.

In Figure 1, we show the flux-calibrated dedispersed S-band dynamic spectrum of the burst B1 using a DM value of $87.77 \mathrm{pc} \mathrm{cm}^{-3}$. In this case, we coherently dedispersed the baseband data to form filterbanks comprised of channelized power spectral densities with temporal and spectral resolutions of $2 \mu$ s and $0.5 \mathrm{MHz}$, respectively. The frequency-averaged burst profile is shown in the upper panel, while the time-averaged on-pulse spectrum is shown in the right panel.

In Figure 2, we show the coherently dedispersed $\left(87.77 \mathrm{pc} \mathrm{cm}^{-3}\right)$ burst time series with $62.5 \mathrm{~ns}$ time resolution. The top panel shows the burst in flux-calibrated total intensity, while the lower panels show the right and left circular polarizations given in units of $\mathrm{S} / \mathrm{N}$. A detailed polarization analysis of B1 and additional bursts from this observation will be presented in a forthcoming publication.

The burst is made up of several remarkably narrow, isolated components, numbering at least seven nonoverlapping components, labeled as $\mathrm{C} 1-\mathrm{C} 7$ in Figure 2. Component $\mathrm{C} 1$ is relatively narrow and appears to be preceded by a short period of low-level emission. This component is followed by a series of similarly narrow components with typical separation of $2-3 \mu \mathrm{s}$. Component $\mathrm{C} 4$ is not resolved temporally with a width that is at least as short as the sampling time of $62.5 \mathrm{~ns}$. The last component, C7, seems to be broader with a width of a few microseconds. We also note the presence of a broad precursor emission in Figure 1, arriving $\sim 80 \mu$ s prior to the arrival time of B1. In Table 1 , we list the peak time, peak $S / N$, $\mathrm{DM}$ value that maximized the peak $\mathrm{S} / \mathrm{N}$, burst width, burst peak flux density, and fluence. We also show in this table the flux density and fluence of the narrowest component of the burst, C4.

Although B1 was detected with high $\mathrm{S} / \mathrm{N}$ at S-band, there was no detectable signal during the same time at X-band. Since no bursts were detected at X-band, we place a $7 \sigma$ upper limit of

\footnotetext{
11 See https://deepspace.jpl.nasa.gov/files/DSN_Radio_Astronomy_Users_ Guide.pdf.
} 


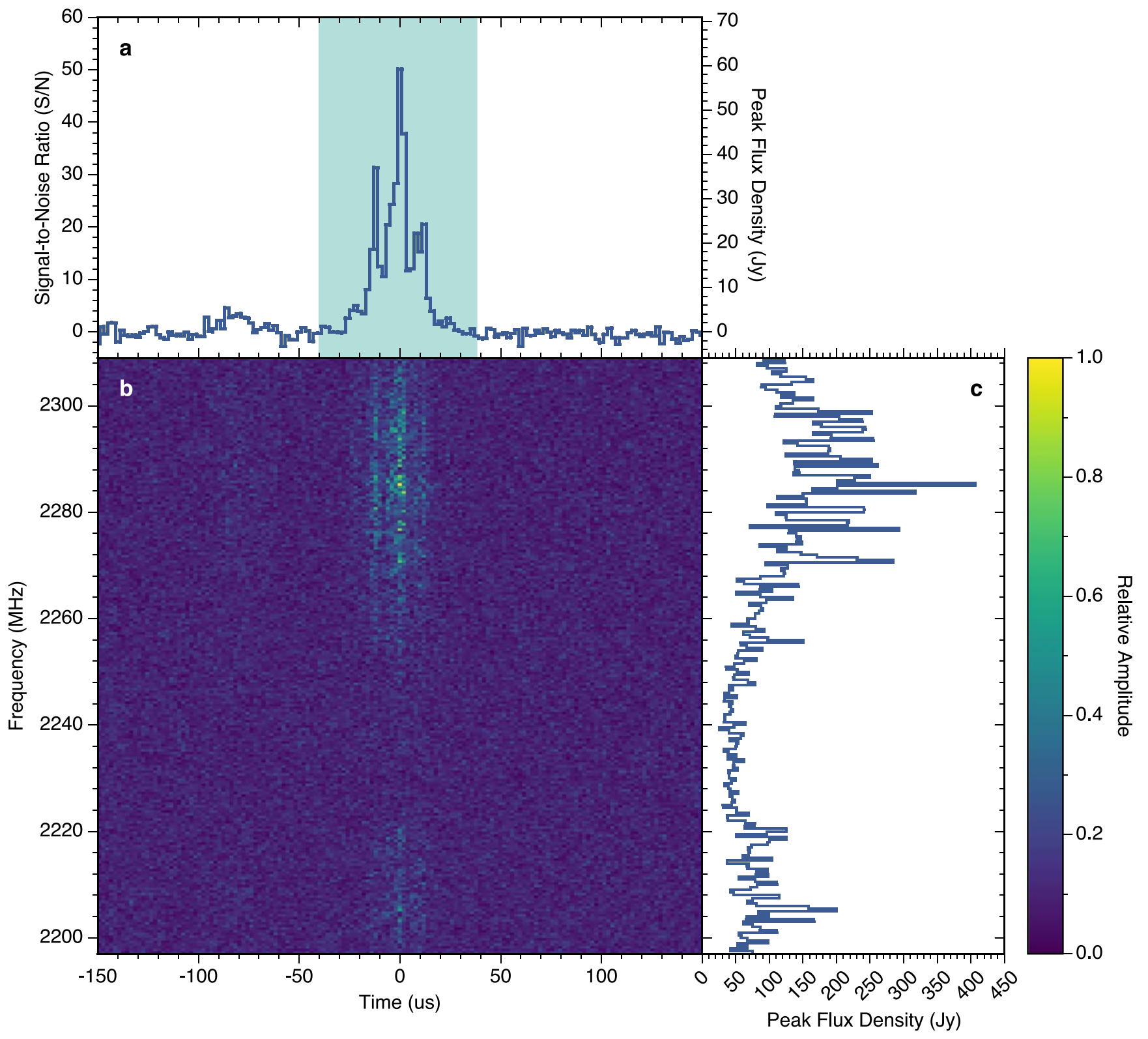

Figure 1. Dynamic spectrum of B1, the brightest S-band burst detected during our observation. The data are plotted with a time and frequency resolution of $2 \mu$ s and $0.5 \mathrm{MHz}$, respectively. The $\mathrm{S} / \mathrm{N}$ maximizing DM $\left(87.77 \mathrm{pc} \mathrm{cm}^{-3}\right)$ was used for dedispersion. Panel (a) shows the frequency-averaged, dedispersed burst profile. The teal shaded region shows the interval that was used for computing the on-pulse spectrum in panel (c). The dedispersed dynamic spectrum is shown in panel (b), and the color bar indicates the relative intensity of the features.

$1.1 \mathrm{Jy}$ on the flux density of the emission at $8.36 \mathrm{GHz}$ during this observation, assuming a similar burst width of $33 \mu \mathrm{s}$. At the narrowest component width $(62.5 \mathrm{~ns})$, corresponding to a single time sample in this observation, the $7 \sigma$ upper limit on the flux density at X-band is $25 \mathrm{Jy}$.

If we further assume that the flux density of FRBs scales as a power law (i.e., $S(\nu) \propto \nu^{\alpha}$, where $S(\nu)$ denotes the flux density at an observing frequency $\nu$ and $\alpha$ is the spectral index), which is typical of most pulsar radio spectra, then we can place an upper limit of $\alpha<-1.3$ on the spectral index of the emission process for the wider burst. On the other hand, using the measured flux density of the narrowest component at S-band and assuming its width at X-band is similarly narrow, we can place an upper limit of $\alpha<-1.8$ on the spectral index of the emission process. However, we note that previous multiwavelength observations of
FRBs show patchy emission over large, instantaneous frequency bandwidths (Majid et al. 2020; Pearlman et al. 2020) and do not appear to support a power-law model (e.g., Scholz et al. 2016; Spitler et al. 2016; Law et al. 2017).

The burst spectrum (Figure 1) shows frequency structure on the order of $10 \mathrm{MHz}$ across the band. Fitting Gaussians to the two bumps in the spectrum, we find full width at half-maximum values of $\mathrm{FWHM}_{1}=39.1 \pm 0.7 \mathrm{MHz}$ at $\nu_{c, 1}=2286.6 \pm 0.3 \mathrm{MHz}$ and $\mathrm{FWHM}_{2}=24 \pm 2 \mathrm{MHz}$ at $\nu_{c, 2}=2207.5 \pm 0.8 \mathrm{MHz}$. This structure could be intrinsic or a result of diffractive interstellar scintillation. The NE2001 electron density model of the Milky Way (Cordes \& Lazio 2020) predicts that our Galaxy will impart a pulse broadening time of $\tau_{\mathrm{sc}, 1}=200 \mathrm{~ns}$ for an extragalactic source along the line of sight to this FRB $\left(\ell=142^{\circ} .2, b=+41^{\circ} .2\right)$. Scaling to $2.3 \mathrm{GHz}$ as $\nu^{-4}$, this gives $\tau_{\mathrm{sc}}=8 \mathrm{~ns}$, well below 

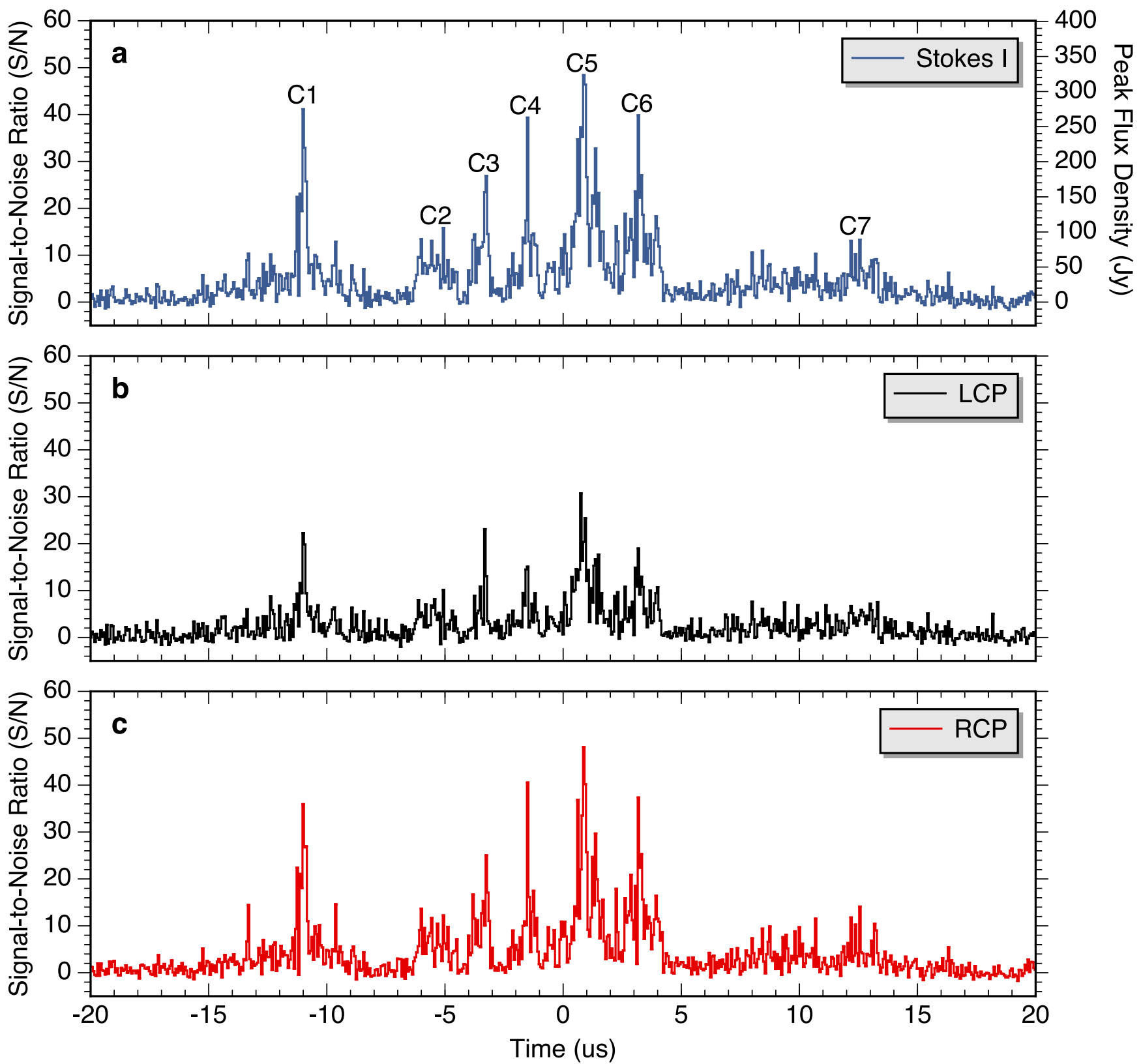

Figure 2. Coherently dedispersed S-band burst profiles shown at $62.5 \mathrm{~ns}$ time resolution, derived from baseband data with sub-bands of $16 \mathrm{MHz}$. In panel (a), we show the Stokes I burst profile, derived from combining the LCP and RCP baseband data. The LCP and RCP burst profiles are shown in panels (b) and (c), respectively. The data have been dedispersed using the $\mathrm{S} / \mathrm{N}$ maximizing DM of $87.77 \mathrm{pc} \mathrm{cm}^{-3}$. This plot shows that the burst is comprised of multiple bright, narrow peaks, with widths of $\lesssim 100 \mathrm{~ns}$. The labels (C1-C7) displayed in panel (a) correspond to seven distinct pulse components comprising the burst.

the time resolution of our data. The scintillation bandwidth corresponding to this scattering time is

$$
\Delta \nu_{\mathrm{DISS}} \sim \frac{1}{2 \pi \tau_{\mathrm{sc}}},
$$

which gives $\Delta \nu_{\text {DISS }} \approx 20 \mathrm{MHz}$ at $2.3 \mathrm{GHz}$. The frequency structure we see in our burst spectrum is entirely consistent with modulation from scintillation, but measurements at other frequencies will be needed to confirm this. We note that the spectrum of B1 is not well modeled by a power law (i.e., $S(\nu) \propto \nu^{\alpha}$, where $S(\nu)$ denotes the flux density at an observing frequency $\nu$ and $\alpha$ is the spectral index). This is also confirmed in previous studies of FRB spectra, where generally the burst spectra are not well modeled by a power-law dependence (e.g.,
Scholz et al. 2016; Law et al. 2017; Majid et al. 2020). If the structure is indeed caused by scintillation, then this could also explain the lack of a detection at $8.36 \mathrm{GHz}$ where the scintillation bandwidth would be $\Delta \nu_{\text {DISS }} \approx 3.5 \mathrm{GHz}$ and we could have $100 \%$ modulations in our band. However, FRBs are typically not broadband, so the lack of a detection at X-band could also be explained by the intrinsic structure of the burst. Observations at other frequencies, particularly instantaneous broadband observations, will confirm whether the frequency structure we see in B1 at S-band along with a simultaneous lack of emission at X-band is indeed the result of scintillation or intrinsic structure of the burst. Similar narrowband emission behavior has also been in seen in FRB 121102 and FRB 180916.J0158 +65, where detections of bright emission at 


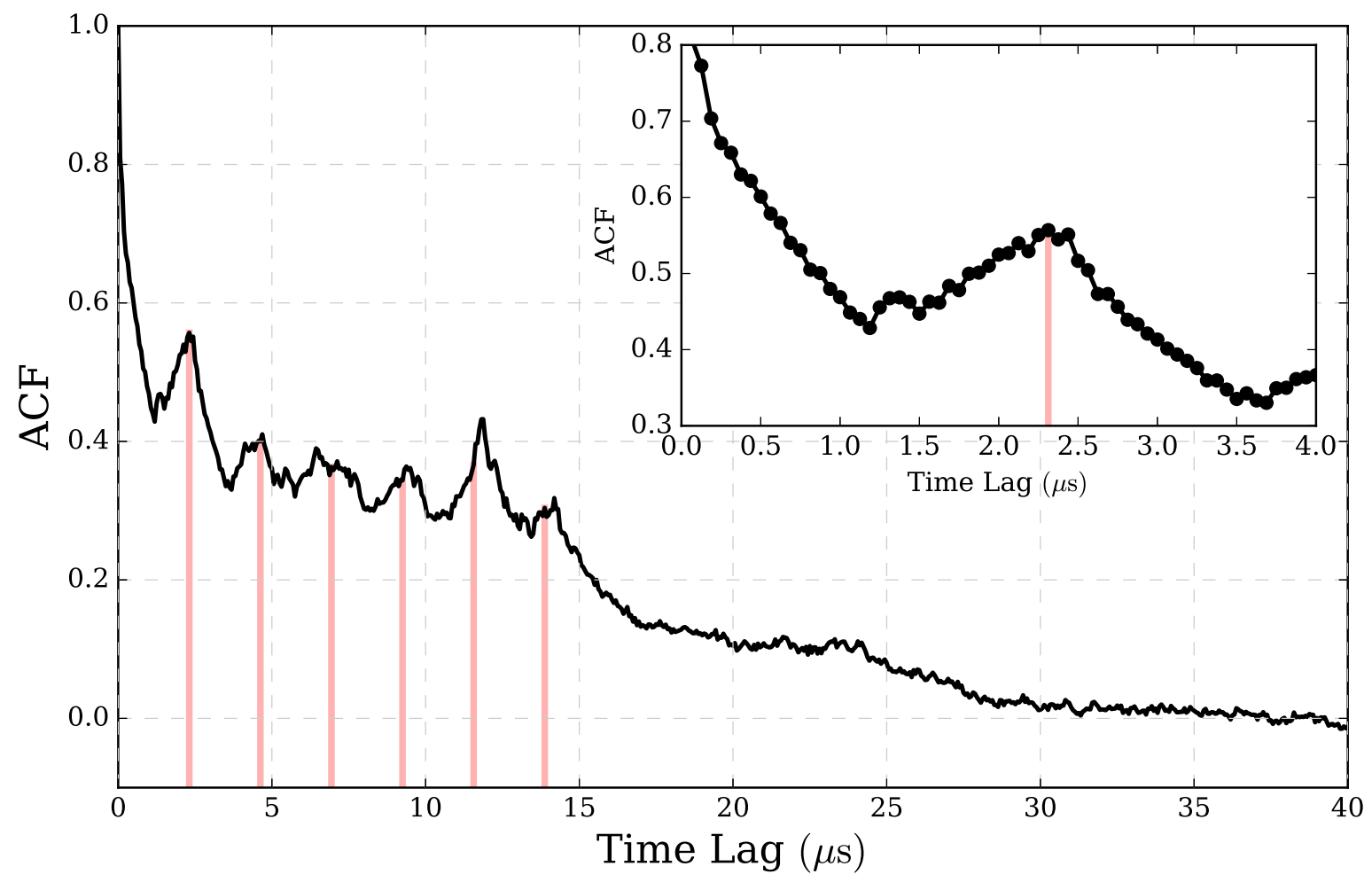

Figure 3. Autocorrelation function of the total intensity burst out to time lags of $40 \mu \mathrm{s}$. At time lags $\lesssim 15 \mu \mathrm{s}$, there are six peaks with roughly equal spacing. The red lines show integer multiples of $2.3 \mu \mathrm{s}$, which is the time lag of the first peak. The last peak is at a lag of $\approx 14 \mu \mathrm{s}$, which corresponds to the time between component 1 and component 6 in Figure 2. The inset shows the ACF at small lags, and the red line indicates the maximum of the first peak.

Table 1

Radio Burst B1 from FRB 20200120E Detected with DSS-63

\begin{tabular}{lc}
\hline \hline Peak Time (MJD) & $59275.99804894308^{\mathrm{a}, \mathrm{b}}$ \\
DM & $87.77 \pm 0.02^{\mathrm{c}}$ \\
Peak S/N & $50.1^{\mathrm{b}, \mathrm{d}}$ \\
Burst Width & $33 \pm 1 \mu \mathrm{s}^{\mathrm{b}, \mathrm{e}}$ \\
Burst Peak Flux Density & $59 \pm 12 \mathrm{Jy}^{\mathrm{b}, \mathrm{f}}$ \\
Burst Fluence & $0.76 \pm 0.15 \mathrm{Jy} \mathrm{ms}^{\mathrm{b}, \mathrm{f}, \mathrm{g}}$ \\
Flux Density of Narrowest Component (C4) & $270 \pm 54 \mathrm{Jy}^{\mathrm{b}, \mathrm{f}}$ \\
Fluence of Narrowest Component (C4) & $0.017 \pm 0.003 \mathrm{Jy} \mathrm{ms}{ }^{\mathrm{f}, \mathrm{g}, \mathrm{h}}$ \\
\hline
\end{tabular}

Notes.

${ }^{\text {a }}$ Barycentric time of arrival (ToA) using the position R.A. $(\mathrm{J} 2000)=$ 09:57:56.7, decl. (J2000) $=68: 49: 32.0$ (Bhardwaj et al. 2021).

${ }^{\mathrm{b}}$ Value is derived from data sampled at a time resolution of $2 \mu \mathrm{s}$.

${ }^{\mathrm{c}} \mathrm{DM}$ value that maximized the peak $\mathrm{S} / \mathrm{N}$ of the burst.

${ }^{\mathrm{d}}$ Peak signal-to-noise ratio $(\mathrm{S} / \mathrm{N})$ after dedispersing with the peak $\mathrm{S} / \mathrm{N}$ maximizing DM of $87.77 \mathrm{pc} \mathrm{cm}^{-3}$.

${ }^{\mathrm{e}}$ Burst width at $10 \%$ maximum.

${ }^{\mathrm{f}}$ Uncertainties are dominated by the $20 \%$ fractional error on the system temperature, $T_{\text {sys. }}$.

${ }^{\mathrm{g}}$ Fluence determined using full width at $10 \%$ of peak SNR. This choice ensures that all of the burst energy is included.

${ }^{\mathrm{h}}$ Value is derived from data sampled at a time resolution of $62.5 \mathrm{~ns}$.

lower frequencies have not been accompanied with emission at higher frequencies during simultaneous observations (Majid et al. 2020; Pearlman et al. 2020; Scholz et al. 2020).

\section{Discussion}

High time resolution studies of FRBs are an important observational tool for discerning proposed emission mechanisms for FRBs, improving our ability to measure fundamental properties of FRBs such as burst energetics, shortest timescales of the emission process that could constrain the spatial sizes of the emission region, and burst energy densities. Furthermore, such high time resolution studies make it possible to carry out detailed studies of scatter broadening due to the turbulent plasma between the source and the observer, including studies of ISM in the Milky Way, the Milky Way halo, host galaxy halo, ISM in the host galaxy, and finally any local scattering material near the progenitors of FRBs. Finally, high time resolution studies of FRBs will also help in identifying known analogs to FRB emission characteristics, such as the giant-pulse phenomenon seen in some pulsars, Type III solar radio bursts, and short S-shaped decametric Jovian bursts (see, e.g., Shaposhnikov et al. 2011; Hankins et al. 2015; Tan et al. 2019).

\subsection{Burst Microstructure}

As shown in Figure 2, B1 is comprised of several narrow $(\lesssim 1 \mu \mathrm{s})$ components contained within a larger burst envelope ( $\sim 33 \mu \mathrm{s})$. In Figure 3, we show the autocorrelation function (ACF) of the total intensity burst B1. The ACF shows correlations out to time lags of $\approx 30 \mu$ s and six peaks at time lags $\lesssim 15 \mu$ s. The peaks in the ACF have widths of 1-2 $\mu$ s and are roughly evenly spaced with separations of $\approx 2.3 \mu \mathrm{s}$. This is consistent with what we see in B1, where the seven components shown in Figure 2 have separations of 1.9-2.7 $\mu$ s (or integer multiples thereof). While the component separations are not strictly periodic in B1, there is clearly a repetition timescale of $\approx 2 \mu \mathrm{s}$ in the burst structure.

The structure seen in $\mathrm{B} 1$ is qualitatively similar to what was seen in the scattering-limited observations of FRB 20180916B (Nimmo et al. 2021a), but on much longer timescales, and is strikingly similar to what is seen in some giant pulses from the 
Crab pulsar (Hankins et al. 2003; Hankins \& Eilek 2007). While the exact emission mechanism may not be the same between FRBs and Crab giant pulses, bursts from both sources are generically expected to be comprised of many individual shots of emission (Cordes \& Wasserman 2016). The small scattering toward FRB 20200120E and the high time resolution of these observations have enabled the clear detection of burst microstructure.

The roughly periodic occurrence of burst components within B1 is also reminiscent of the microstructure seen in single pulses of some radio pulsars. Pulsar microstructure describes the rapid intensity fluctuations within individual single pulses. $\mathrm{ACF}$ analyses like the one performed here have revealed that the microstructure of some pulsars has recurring periodicities (e.g., Cordes et al. 1990; Lange et al. 1998). While not seen in every radio pulsar, microstructure periodicities have been measured in several slow spinning pulsars (Lange et al. 1998; Mitra et al. 2015), the young Vela pulsar (Kramer et al. 2002), and even millisecond pulsars (De et al. 2016). Interestingly, there is a correspondence between the microstructure periodicity $\left(P_{\mu}\right)$ and the pulsar spin period $\left(P_{\text {spin }}\right)$ given by

$$
P_{\mu} \sim 10^{-3} P_{\text {spin }}
$$

albeit with significant scatter (Mitra et al. 2015; De et al. 2016).

While it is not known if the origin of pulsar microstructure is geometric (beam sweeping past) or temporal modulation, the phenomenological similarity between $\mathrm{B} 1$ and periodic microstructure in pulsars is tantalizing and may suggest a magnetospheric origin for FRB 20200120E. If the $P_{\mu} \approx 2-3 \mu$ s structure seen in B1 follows the $P_{\mu}-P_{\text {spin }}$ relation for pulsar microstructure, it would imply a spin period of several milliseconds, which could be achieved by a fast-spinning young pulsar or a recycled millisecond pulsar. The latter would be consistent with the localization of this FRB to a globular cluster (Kirsten et al. 2021a), which tends to be fertile grounds for the formation of millisecond pulsars.

\subsection{Luminosity and Brightness Temperature}

Figure 4 shows how the full burst B1 and its narrowest component $\mathrm{C} 4$ compared to other short duration radio pulses in the time-luminosity phase space. If we assume that the burst source is at the same distance as M81 $(d=3.6 \mathrm{Mpc})$, then B1 is on the faint end of the FRB luminosity distribution with a pseudoluminosity of $S_{\mathrm{pk}} d^{2} \approx 8 \times 10^{8} \mathrm{Jy} \mathrm{kpc}^{2}$ and an isotropic equivalent spectral luminosity of $L_{\nu, \text { iso }}=4 \pi S_{\mathrm{pk}} d^{2} \approx 9 \times 10^{29} \mathrm{erg} \mathrm{s}^{-1} \mathrm{~Hz}^{-1}$, which is only a factor of $\approx 3$ larger than the SGR $1935+2154$ burst detected by STARE2 (Bochenek et al. 2020). Continued monitoring of this FRB with large telescopes will characterize the faint end of the FRB luminosity distribution to levels below the STARE2 burst.

The narrowest component, $\mathrm{C} 4$, has a pseudoluminosity of $S_{\mathrm{pk}} d^{2} \approx 3.5 \times 10^{9} \mathrm{Jy} \mathrm{kpc}{ }^{2}$ and an isotropic equivalent spectral luminosity of $L_{\nu \text {,iso }} \approx 4 \times 10^{30} \mathrm{erg} \mathrm{s}^{-1} \mathrm{~Hz}^{-1}$. This is only $\approx 380$ times larger than the Crab nanoshot $(S=2.3 \mathrm{MJy}, W=0.4 \mathrm{~ns}$, $d=2 \mathrm{kpc}$ ) seen by Hankins \& Eilek (2007) and occupies the sparsely populated short duration region of the time-luminosity phase space (Figure 4). The brightness temperature, given by

$$
T_{\mathrm{b}}=\frac{S d^{2}}{2 k_{\mathrm{B}}(\nu W)^{2}}
$$

is $T_{\mathrm{b}} \approx 2 \times 10^{40} \mathrm{~K}$ for component $\mathrm{C} 4$, which is one of the highest measured $T_{\mathrm{b}}$ values for any FRB and only a factor of $\approx 10$ smaller than the Crab nanoshot (Hankins \& Eilek 2007). Of course, since this narrow component is still unresolved in time, the true brightness temperature may be much larger.

\subsection{Size of the Emission Region}

Farah et al. (2018) reported the discovery of FRB 170827, which shows complex temporal structure with components as short as $\sim 30 \mu \mathrm{s}$, indicating that the emission region may be as small as only $10 \mathrm{~km}$. Similarly, Cho et al. (2020) showed the presence of microstructure in their study of FRB 181112, resolving emission components as short as $15 \mu \mathrm{s}$, implying emission size of a few kilometers. Nimmo et al. (2021a) reported on high time resolution observations of FRB 20180916B, where they observed several bursts with a range of timescales from a few milliseconds to as short as a few microseconds wide. Their observations, carried out at $1.7 \mathrm{GHz}$, were limited in resolving narrower structures by multipath scattering. They attribute the observation of 3-4 $\mu$ s structure in one of the detected bursts to an emission region on the order of $1 \mathrm{~km}$.

We are reporting a significantly shorter variability timescale for FRB 20200120E than for any previously published FRB. Very recently Nimmo et al. (2021b) also reported similar timescale emission for FRB 20200120E. While the envelope of emission spans $\sim 30 \mu \mathrm{s}$, the burst is clearly comprised of distinct and nonoverlapping narrow components. Using a light travel time argument, the short duration of the individual components imply small source sizes $\sim c \delta t$, where $\delta t$ is the width of individual components. For an emission timescale of $\sim 100 \mathrm{ns,} \mathrm{corresp-}$ onding to the width of the narrowest component, the size of the emission region is constrained to be $r \lesssim 30 \mathrm{~m}$.

However, relativistic effects can significantly alter the relation between size of the emission region and the variability timescale. For instance, Beniamini \& Kumar (2020) consider the case of a relativistic outflow moving with a Lorentz factor $\gamma$ producing a radio burst at distance $R$ from the origin of the outflow, and define a characteristic timescale, $t_{0}$,

$$
t_{0}(R) \equiv \frac{R}{2 c \gamma^{2}} \sim 70 \mathrm{~ns}\left(\frac{R}{10^{9} \mathrm{~cm}}\right)\left(\frac{\gamma}{500}\right)^{-2},
$$

where the scaling has been chosen to give a timescale comparable to the shortest timescale observed in the burst reported here. Large $\gamma$ or relatively small $R$ are required to explain variability timescales with $t_{0} \lesssim 100 \mathrm{~ns}$. As discussed by Beniamini \& Kumar (2020), the variability timescale may be even shorter than the characteristic timescale given in Equation (4) due to a number of possible factors: clumps in the emission region, a narrow range of emission radii, radial evolution of the spectrum, or anisotropic emission. Given our observation that the burst components appear to have comparable rise and fall times, clumpiness or anisotropic emission would be favored over narrow emission radii or radial spectral evolution. In any case, as Beniamini \& Kumar (2020) point out, the efficiency of conversion of energy from the relativistic outflow must decrease when a physical mechanism is invoked to decrease the timescale of variability.

\subsection{Energy Density}

Using the light travel time estimate, and combining the size of the emission region with the measured fluence (Table 1), we estimate the energy spectral density of the component to be 


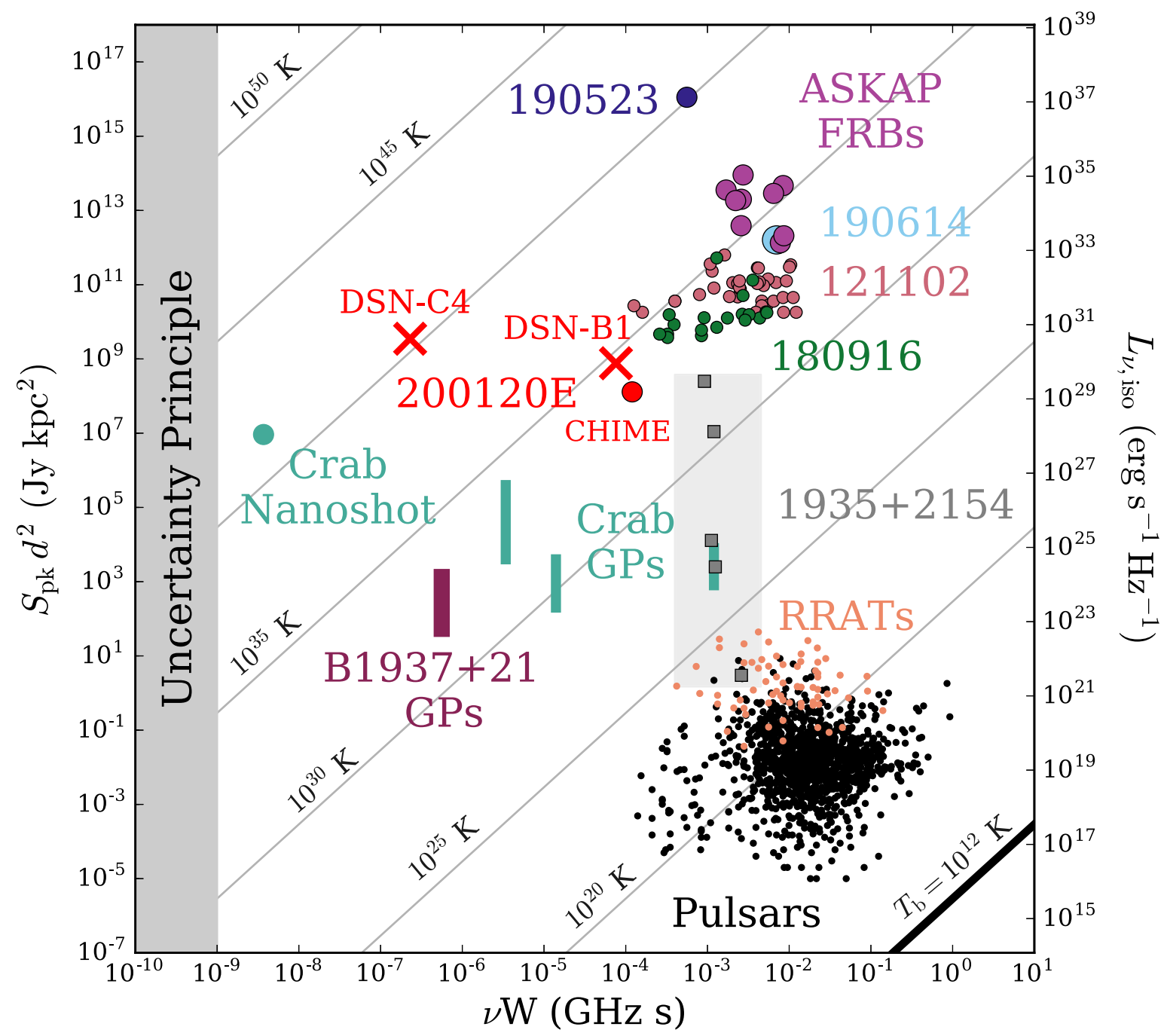

Figure 4. Time-luminosity phase-space plot for radio bursts showing the product of observing frequency and pulse width ( $\nu W$ in GHz s) against the pseudoluminosity $\left(S_{\mathrm{pk}} d^{2}\right.$, left $)$ and equivalent isotropic spectral luminosity $\left(L_{\nu, \text { iso }}=4 \pi S_{\mathrm{pk}} d^{2}\right.$, right). The brightness temperature $T_{\mathrm{b}}=\left(1 / 2 k_{\mathrm{b}}\right) \times\left(S d^{2}\right) /(\nu W)^{2}$ is indicated by black lines with the nominal limit for coherent emission $\left(T_{\mathrm{b}} \sim 10^{12} \mathrm{~K}\right)$ shown as a thick black line. The shaded gray region at $\nu \mathrm{W} \lesssim 10^{-9} \mathrm{GHz} \mathrm{s}$ is excluded due to the uncertainty principle. Pulsars (Manchester et al. 2005) and RRATs with known distances are shown in black and orange dots, respectively. Giant pulses from the Crab pulsar (Lundgren et al. 1995; Karuppusamy et al. 2010; Majid et al. 2011) and B1937 + 21 (McKee et al. 2019) are shown as green and purple lines, respectively, that indicate the range of pulse flux densities observed. The $0.4 \mathrm{~ns}$ Crab "nanoshot" (Hankins \& Eilek 2007) is shown as a green dot. Bursts from the magnetar SGR $1935+2154$ are shown as gray squares in a shaded gray box that indicates the extent of observed emission (Bochenek et al. 2020; CHIME/FRB Collaboration et al. 2020; Zhang et al. 2020; Kirsten et al. 2021b). FRBs with host galaxies with measured redshifts are also shown and labeled. For the repeaters FRB 121102 (Scholz et al. 2016; Spitler et al. 2016; Gajjar et al. 2018) and FRB 180916 (Marthi et al. 2020; Marcote et al. 2020), multiple bursts are shown. For FRB 200120E, we show the initial CHIME detection as a red circle (Bhardwaj et al. 2021), and our results as red crosses. DSN-B1 is the full $33 \mu$ s burst and DSN-C4 is the narrowest ( $\sim 100 \mathrm{~ns})$ component of the burst.

$0.15 \times 10^{13} \mathrm{~J} \mathrm{~m}^{-3} \mathrm{~Hz}^{-1}$ in the rest frame of the emitting source. Under the conservative assumption that the emission is confined only to our observing band, we estimate the total energy density of the component to be $1.6 \times 10^{20} \mathrm{~J} \mathrm{~m}^{-3}$. If we attribute the entire energy density to the equivalent energy density in a magnetic field $u_{B}=B^{2} / 2 \mu$, where $B$ is the magnetic field and $\mu$ is the magnetic permeability, we obtain a magnetic field $B \sim 2 \times 10^{11} \mathrm{G}$. We note that if relativistic effects such as beaming are taken into account (see the discussion in Section 4.3 above) the estimated magnetic field required to serve as the emission reservoir can be significantly lower than the value estimated above.

\subsection{Propagation Effects}

The extremely high time resolution of our data allows us to set a pulse broadening limit of $\tau_{\mathrm{sc}} \lesssim 100 \mathrm{~ns}$ to the total $2.3 \mathrm{GHz}$ scattering along the line of sight to FRB 20200120E. Scaling to $1 \mathrm{GHz}$ as $\nu^{-4}$, this corresponds to a limit of $\tau_{\mathrm{sc}, 1 \mathrm{GHz}} \lesssim 3 \mu \mathrm{s}$. A small scattering time is to be expected since the line of sight $\left(\ell=142^{\circ} .2, b=+41^{\circ} .2\right)$ only passes through a small part of the Milky Way and the FRB likely resides in the outer edges of M81.

The NE2001 electron density model (Cordes \& Lazio 2020) predicts a Milky Way contribution to the $2.3 \mathrm{GHz}$ pulse broadening time of $\tau_{\mathrm{sc}}=8 \mathrm{~ns}$ at $2.3 \mathrm{GHz}$ along the line of sight to the FRB. Although this is well below our time resolution limit, the corresponding scintillation bandwidth is $\Delta \nu_{\text {DISS }} \approx 20 \mathrm{MHz}$, which is entirely consistent with the frequency structure we see in B1. If there is any additional scattering caused by the host galaxy or the Milky Way halo (which is not modeled in NE2001), it would be limited to $\tau_{\text {sc }} \lesssim 100 \mathrm{~ns}$, which would correspond to a scintillation bandwidth of $\Delta \nu_{\text {DISS }} \gtrsim 2 \mathrm{MHz}$ at $2.3 \mathrm{GHz}$. We see 
no obvious signs of correlations on frequency separations less than $\sim 10 \mathrm{MHz}$, which suggests that any additional scattering is smaller than the contribution from the Milky Way disk. This is consistent with the apparent location of the FRB outside M81 (Bhardwaj et al. 2021) and the small amount of scattering expected from the Milky Way halo (Ocker et al. 2021).

Observations at other frequencies will confirm whether the frequency structure we see in B1 is indeed the result of scintillation. Since the line of sight appears to be relatively uncontaminated by both the host and our own Galaxy, this FRB could be a very nice source for probing the properties of the ionized gas in the Galactic halo. Furthermore, the very small scattering seen here $\left(\tau_{\text {sc }} \lesssim 100 \mathrm{~ns}\right)$ means that even at $100 \mathrm{MHz}$ the scattering would be $\tau_{\mathrm{sc}, 0.1} \lesssim 30 \mathrm{~ms}$. We therefore strongly encourage low frequency observations of FRB 20200120E for probing the Galactic halo.

\subsection{Future Outlook}

With the detection of unresolved burst structure at the native time resolution $(62.5 \mathrm{~ns})$ during the observations reported here, the question remains whether the individual components in bursts from FRB 20200120E are even narrower in width at nanosecond timescales. Such studies are best carried out at higher radio frequencies, where the effects of multipath scatter broadening are mitigated. Since the temporal width of the bursts is a fundamental observational property of the emission processes and has important implications for determining the burst energetics, we encourage future observations of FRBs with even higher temporal resolutions at higher radio frequencies. On the other hand, as mentioned in the previous section, FRB 20200120E also offers an opportunity to study the properties of the ionized medium in the halo of the the Milky Way. Low frequency observations, where the effects of multipath scattering are amplified, would also be quite useful for this purpose.

We thank Paz Beniamini and Sterl Phinney for informative discussions during the preparation of this paper.

A.B.P is a McGill Space Institute (MSI) Fellow and a Fonds de Recherche du Quebec-Nature et Technologies (FRQNT) postdoctoral fellow. R.S.W. is supported by an appointment to the NASA Postdoctoral Program at the Jet Propulsion Laboratory, administered by Universities Space Research Association under contract with NASA. M.B. is supported by an FRQNT Doctoral Research Award. C.L. was supported by the U.S. Department of Defense (DoD) through the National Defense Science and Engineering Graduate Fellowship (NDSEG) Program. E.P. acknowledges funding from an NWO Veni Fellowship.

We thank the Jet Propulsion Laboratory's and California Institute of Technology's President's and Director's Research and Development Fund for supporting this work. We also thank Dr. Charles Lawrence and Dr. Stephen Lichten for providing programmatic support. In addition, we are grateful to the DSN scheduling team (Hernan Diaz, George Martinez, Carleen Ward), the Madrid Deep Space Communication Complex (MDSCC) staff for scheduling and carrying out these observations, and Steven Olson for his help in flux calibration of the data.

A portion of this research was performed at the Jet Propulsion Laboratory, California Institute of Technology and the Caltech campus, under a Research and Technology Development Grant through a contract with the National Aeronautics and Space Administration. U.S. government sponsorship is acknowledged.

\section{ORCID iDs}

Walid A. Majid (ib https://orcid.org/0000-0002-4694-4221

Aaron B. Pearlman (i) https://orcid.org/0000-0002-8912-0732 Thomas A. Prince (i) https://orcid.org/0000-0002-8850-3627 Robert S. Wharton (iD https://orcid.org/0000-0002-7416-5209 Charles J. Naudet (ib https://orcid.org/0000-0001-6898-0533

Karishma Bansal (i) https://orcid.org/0000-0002-7418-7862

Liam Connor (i) https://orcid.org/0000-0002-7587-6352

Mohit Bhardwaj (D) https://orcid.org/0000-0002-3615-3514

Shriharsh P. Tendulkar (iD https://orcid.org/0000-0003-

2548-2926

\section{References}

Bannister, K. W., Deller, A. T., Phillips, C., et al. 2019, Sci, 365, 565 Beniamini, P., \& Kumar, P. 2020, MNRAS, 498, 651

Bhardwaj, M., Gaensler, B. M., Kaspi, V. M., et al. 2021, ApJL, 910, L18

Bochenek, C. D., Ravi, V., Belov, K. V., et al. 2020, Natur, 587, 59

Chatterjee, S., Law, C. J., Wharton, R. S., et al. 2017, Natur, 541, 58

CHIME/FRB Collaboration, Andersen, B. C., Bandura, K. M., et al. 2020, Natur, 587, 54

Cho, H., Macquart, J.-P., Shannon, R. M., et al. 2020, ApJL, 891, L38

Cordes, J. M., \& Chatterjee, S. 2019, ARA\&A, 57, 417

Cordes, J. M., \& Lazio, T. J. W. 2020, arXiv:astro-ph/0207156

Cordes, J. M., \& Wasserman, I. 2016, MNRAS, 457, 232

Cordes, J. M., Weisberg, J. M., \& Hankins, T. H. 1990, AJ, 100, 1882

De, K., Gupta, Y., \& Sharma, P. 2016, ApJL, 833, L10

Farah, W., Flynn, C., Bailes, M., et al. 2018, MNRAS, 478, 1209

Fonseca, E., Andersen, B. C., Bhardwaj, M., et al. 2020, ApJL, 891, L6

Gajjar, V., Siemion, A. P. V., Price, D. C., et al. 2018, ApJ, 863, 2

Hankins, T. H., \& Eilek, J. A. 2007, ApJ, 670, 693

Hankins, T. H., Jones, G., \& Eilek, J. A. 2015, ApJ, 802, 130

Hankins, T. H., Kern, J. S., Weatherall, J. C., \& Eilek, J. A. 2003, Natur, 422, 141

Karuppusamy, R., Stappers, B. W., \& van Straten, W. 2010, A\&A, 515, A36

Kirsten, F., Marcote, B., Nimmo, K., et al. 2021a, arXiv:2105.11445

Kirsten, F., Snelders, M. P., Jenkins, M., et al. 2021b, NatAs, 5, 414

Kramer, M., Johnston, S., \& van Straten, W. 2002, MNRAS, 334, 523

Lange, C., Kramer, M., Wielebinski, R., \& Jessner, A. 1998, A\&A, 332, 111

Law, C. J., Abruzzo, M. W., Bassa, C. G., et al. 2017, ApJ, 850, 76

Lundgren, S. C., Cordes, J. M., Ulmer, M., et al. 1995, ApJ, 453, 433

Majid, W. A., Naudet, C. J., Lowe, S. T., \& Kuiper, T. B. H. 2011, ApJ, 741,53

Majid, W. A., Pearlman, A. B., Nimmo, K., et al. 2020, ApJL, 897, L4

Manchester, R. N., Hobbs, G. B., Teoh, A., \& Hobbs, M. 2005, AJ, 129, 1993

Marcote, B., Nimmo, K., Hessels, J. W. T., et al. 2020, Natur, 577, 190

Marthi, V. R., Gautam, T., Li, D. Z., et al. 2020, MNRAS, 499, L16

McKee, J. W., Stappers, B. W., Bassa, C. G., et al. 2019, MNRAS, 483, 4784

Mitra, D., Arjunwadkar, M., \& Rankin, J. M. 2015, ApJ, 806, 236

Nimmo, K., Hessels, J. W. T., Keimpema, A., et al. 2021a, NatAs, 5, 594

Nimmo, K., Hessels, J. W. T., Kirsten, F., et al. 2021b, arXiv:2105.11446

Ocker, S. K., Cordes, J. M., \& Chatterjee, S. 2021, ApJ, 911, 102

Pearlman, A. B., Majid, W. A., Prince, T. A., et al. 2020, ApJL, 905, L27

Petroff, E., Barr, E. D., Jameson, A., et al. 2016, PASA, 33, e045

Petroff, E., Hessels, J. W. T., \& Lorimer, D. R. 2019, A\&ARv, 27, 4

Platts, E., Weltman, A., Walters, A., et al. 2019, PhR, 821, 1

Prochaska, J. X., Macquart, J.-P., McQuinn, M., et al. 2019, Sci, 366, 231

Ravi, V., Catha, M., D’Addario, L., et al. 2019, Natur, 572, 352

Scholz, P., Bogdanov, S., Hessels, J. W. T., et al. 2017, ApJ, 846, 80

Scholz, P., Cook, A., Cruces, M., et al. 2020, ApJ, 901, 165

Scholz, P., Spitler, L. G., Hessels, J. W. T., et al. 2016, ApJ, 833, 177

Shaposhnikov, V. E., Korobkov, S. V., Rucker, H. O., et al. 2011, JGRA, 116, A03205

Spitler, L. G., Scholz, P., Hessels, J. W. T., et al. 2016, Natur, 531, 202

Tan, B., Chen, N., Yang, Y.-H., et al. 2019, ApJ, 885, 90

The CHIME/FRB Collaboration, Amiri, M., Bandura, K., et al. 2019a, Natur, 566,235

The CHIME/FRB Collaboration, Andersen, B. C., Bandura, K., et al. 2019b, ApJL, 885, L24

Zhang, C. F., Jiang, J. C., Men, Y. P., et al. 2020, ATel, 13699, 1 